\title{
Using In-Home Displays to Provide Smart Meter Feedback about Household Electricity Consumption: A Randomized Control Trial Comparing Kilowatts, Cost, and Social Norms
}

\author{
P. Wesley Schultz, California State University San Marcos \\ Mica Estrada, University of California, San Francisco \\ Joseph Schmitt, Action Research \\ Rebecca Sokoloski, California State University San Marcos \\ Nilmini Silva-Send, University of San Diego
}

Author's Note: Address correspondences to Wesley Schultz, Department of Psychology, California State University, San Marcos, CA, 92078.wschultz@ csusm.edu.

The research reported in this paper was funded by a grant from the National Science Foundation (DUE-1239797). The project was part of the Climate Education Partnership at the University of San Diego, led by Michel Boudrias, Mica Estrada, Sasha Gershunov, and Nilmini Silva-Send. The implementation was conducted with a large team at CSUSM, including Anela Amba-Pascua, Maria Aguilar, Andrea Briseño, Stefano De Dominicis, Mariah Parvizi, Nicholas Roome, Perla Sandoval, Sierra Schultz, Kayla Sinfield, Jenna Szuch, and Danielle Teece. Also Valissa Middleton from CSUSM mail services.

We also want to acknowledge the support of San Diego Gas and Electric (SDG\&E), and the staff including Tyler Sybert, Tishmari Lewis, and Nenita Plorin. The in-home displays used in the reported experiment were developed by Rainforest Automation, and we acknowledge the help of Chris Tumpach. 


\begin{abstract}
A randomized control field experiment is reported using in-home displays to reduce household electricity consumption. Custom-coded in-home displays (IHDs) were created to provide real-time household electricity consumption feedback, and were framed as either (a) simple $\mathrm{kW}$ consumption, or (b) $\mathrm{kW}$ consumption and the corresponding cost, or (c) $\mathrm{kW}$ consumption and a dynamically-derived social normative frame. Analyses focused on household electricity consumption in the first week following deployment, and again over a 3-month time span. Findings showed that households receiving simple feedback, and feedback framed as cost did not differ significantly from the randomized control at either the 1-week or the 3-month time points. Similarly, results showed that educational materials alone did not reduce electricity consumption. However, significant effects were found for households receiving the normative frame, which consumed $9 \%$ less than control households during the initial 1-week evaluation period, and 7\% less during the full 3-month evaluation period. Yet despite these findings, residents reported more positive experiences and more obligations to conserve electricity with the cost and feedback IHDs. The results suggest that in-home displays offer promise for encouraging energy conservation, but careful consideration should be given to the way that the feedback is framed.
\end{abstract}




\section{Introduction}

Recent advances in metering infrastructure have afforded new opportunities for communications and feedback aimed at promoting energy efficiency. While high resolution metering has been available for many decades, it is only recently that this technology has been widely deployed. Advanced Metering Infrastructure (AMI)—commonly referred to as smart meters - monitor and record electricity consumption at a high-frequency resolution, most commonly in 1-hour intervals, but resolutions of 15-minute and even near-real-time are also possible. AMI technology is being widely deployed around the world, but surprisingly there are few peer-reviewed reports testing the impact of smart meter enabled feedback on residential electricity consumption. This paper reports a randomized control trial using in-home displays (IHDs) that provide smart meter enabled feedback in a near-real-time context. The experiment also compares the impact of feedback alone, with feedback framed as cost, or as a social norm comparison.

Background. As of 2012, there were an estimated 36 million smart meters deployed in the United States, with a projection of 65 million by the end of 2015 [1]. Worldwide, deployment of smart meters has grown sharply since 2010, and industry estimates suggest that in 2014 there were 100 million smart meters shipped, up from 87 million in 2013 [2]. Among the justifications for purchasing and deploying smart meters are reduced labor costs associated with automated billing, improved billing accuracy, increased energy efficiency, and increased customer engagement $[2,3]$.

This paper focuses on the role of smart meters in promoting more efficient uses of electricity that ultimately reduces consumption. Smartgrid technologies allow for a range of communication opportunities that encourage consumers to modify their behavior, including 
enhanced demand response programs, time of use pricing, smart automation, and high resolution energy feedback [4]. Feedback in particular is often cited as a gateway to efficiency and reduced consumption, and real-time feedback is typically viewed as more influential than less granular forms of feedback such as monthly utility bills (even when the monthly bills are enhanced using AMI data; $[5,6])$.

Feedback. Research on feedback has clearly established that it can promote changes in behavior and assist an individual in achieving a goal [7,8]. With regard to residential electricity consumption, there is considerable evidence showing that feedback can play a role in reducing consumption [9-12]. However the effectiveness of feedback to reduce consumption varies widely across studies, and a number of researchers have concluded that feedback alone is generally not sufficient to induce conservation [13-15]. In fact, in some cases feedback can lead to boomerang effects, resulting in increased levels of consumption [16-18].

Given that feedback can result in a variety of outcomes, it is useful to examine the conditions under which it works best. Froehlich [19] offered a framework for designing effective feedback designs, which included:

- Higher frequency feedback systems to allow the individual to connect their actions with the consequences;

- Meaningful units;

- Highly granular with regard to time, space, source, or category;

- Always available, rather than only upon request.

In addition to these practical recommendations, feedback theory suggests that it is more effective at changing behavior when it is accompanied by a goal. In essence, feedback can help an individual to achieve a goal, but in the absence of a goal, feedback is unlikely to motivate a 
change in behavior $[8,14]$. Goals provide a motivation for action and can originate from the attitudes or values of the person, from a stated commitment, or a social obligation, among others. Two goals in particular have been studied in the context of energy efficiency: saving money, and social norm comparisons. With regard to saving money, many feedback strategies show consumption paired with the cost of the consumption. In fact, the energy efficiency area is replete with examples of cost message frames, but there is some evidence to question their effectiveness [20]. Note that the lack of evidence in this case pertains to the framing of the feedback regarding the behavior's cost, and not changing the cost itself. The latter-changing the cost — has a considerable amount of evidence showing the effects of price elasticity on electricity consumption $[21,22]$.

In recent years, social norms have emerged as a promising alternative message frame. Here, feedback is presented in comparison to similar others, or nearby households, and consumption rates above the norm are identified as deviant, whereas consumption rates below the norm are praised. The basic approach emerged from behavioral science research [17], and has become an industry standard through large-scale applications such as OPOWER [23]. However to date, normative comparisons have been provided based on monthly consumption rates, and to our knowledge, no published papers have examined the potential for a normative feedback message frame in the context of smart meters.

The current study tested the impact of real-time feedback on residential electricity consumption. In recent years, there have been a large number of utility-based pilot programs to study the impact of IHDs, although only a few have used a randomized control design. Faruqui, Sergici, and Sharif [24] reviewed the findings from 12 utility-based pilot programs. Across the 12 pilots, IHDs were associated with reductions in consumption by $7 \%$, but the effects were 
heterogeneous, and several moderating variables were identified. Most relevant to the current study is a pilot program by Canada's Hydro One. The pilot compared households that received an IHD with households that were switched to a time-of-use (TOU) pricing plan, households that received both, and households that received neither (control group). The results showed a reduction of $5.5 \%$ for households with the combined IHD and TOU pricing, but only $1.8 \%$ reduction for households with only the IHD.

In the current study, we focus on how the message frame impacts the effectiveness of feedback provided through IHDs. Feedback was provided to households using an IHD, and a custom coded interface. The devices were provided to a sample of utility customers for free, and households were randomly assigned to receive one of three message frames: kilowatts, cost, or norm comparison. Based on prior research, we hypothesized that the kilowatt feedback alone would not be sufficient to reduce consumption, relative to a randomized control group. Similarly, we hypothesized that the cost message frame would not produce less consumption, compared to a randomized control. Third, we hypothesized that households in the norm message frame would significantly reduce their consumption. We further hypothesized that the norm frame effect would be largest for high-consuming households. Finally, based on prior research [20], we hypothesized that participants would report liking the cost frame more than the kilowatt frame, and that they would report liking the norm frame less than the kilowatt frame. An additional set of analyses was conducted to test for moderating variables of the three message frames.

\section{Method}

This study was conducted in cooperation with a large local investor-owned utility in Southern California. Recruitment for the study was conducted in October 2012, the materials were deployed in June 2013, and household electricity consumption was monitored through the 
following October of 2013. Prior to the launch of the study, smart meters had been installed in all homes for approximately one year.

\section{Participants}

Participants in this study were residents of 431 single-family households. On average, each household had 3.43 residents with a length of residency in the home of 11 years. Regarding income, $80 \%$ reported earning more than $\$ 55,000$ per year (the identified regional median). Politically, 34\% of participants self-identified as Republican, 39\% Democrat, and 27\% other. The single-family homes ranged from 870 to 4400 square feet, with an average size of 2127 square feet. Households with electricity producing solar panels were excluded from this study due to variability in the energy used from the utility. During the two weeks prior to the intervention, the 431 households used an average of $23.83 \mathrm{kWh}$ per day.

\section{Materials}

Pre survey. Households were recruited for the study using a postal mail survey of the identified neighborhoods in the region. Survey items included:

- Climate change knowledge (3 items rated on a scale from 1-7; for example, "How true is the following statement, The rise in carbon dioxide in the atmosphere is a major cause of temperatures rising globally")

- Knowledge of electricity consumption (4 items rated on a scale from 1-7; for example, “How much do you know about electricity consumption in your home?”)

- Obligation to use less electricity (four items on a scale from 1-7; for example, "I feel personally obligated to use less electricity")

- Demographics such as length of time in the home and political affiliation (7 items). 
Pre survey consent. To be included in the study, participants signed a consent form at the end of the survey indicating their interest in participating in the study. The consent form also authorized the research team to obtain the customer's hourly electricity consumption directly from the local utility. The consent authorized data access to the year prior to and up to a year following the study start date.

In-home displays. In cooperation with Rainforest Automation, three different customcoded in-home displays (IHDs) were developed. Each IHD had a digital display and three LED lights (red, yellow, and green). All IHDs used ZigBee technology to wirelessly connect with the smart meter of the participant's home. This wireless connection allowed for household kW consumption to be displayed on the IHD in near real time (approximately 5 second delay). In the remainder of the paper, we use $\mathrm{kW}$ to refer to the instantaneous rate of consumption, and $\mathrm{kWh}$ to refer to the amount of electricity consumed in a 1-hour span. With this distinction, a household that uses $1 \mathrm{~kW}$ consistently for a 1 -hour period would consume $1 \mathrm{kWh}$, but importantly the instantaneous $\mathrm{kW}$ rate can fluctuate widely during a 1-hour time period.

Feedback only. The feedback only IHD was coded to display the household's current kW consumption. The lights were coded as a self-comparison; if the household was using more than its average in the past three minutes the light would be red. Similarly, green indicated the household was using less, and yellow indicated the household was using the same amount of electricity as three minutes prior. To prevent constant switching of lights, a $30 \%$ threshold was set. Therefore, the light would only switch to red from yellow if the household was consuming $30 \%$ more than the average consumption in the past three minutes. See Figure 1 for an image of the feedback only device. 
Cost+feedback. The cost+feedback IHD was coded to display current $\mathrm{kW}$ consumption, along with its associated cost to the participant. The display reflected the instantaneous cost for the level of consumption. The lights were coded to respond to usage in the same way as the feedback only device, with green indicating reduced consumption, yellow steady, and red increased consumption. See Figure 1 for an image of the cost + feedback device. At the time of the study, electricity pricing was based on a 4-tier summer rate, with the highest tier priced at 36cents per kilowatt hour. The devices were coded with the tiered pricing structure, and showed the instantaneous rate for the current tier. Typically, the displayed rates ranged from $.5 \mathrm{~kW}$ (displayed at 18-cents per hour for households in the highest tier) to $1.5 \mathrm{~kW}$ (displayed at 54cents per hour), although substantially higher $\mathrm{kW}$ rates were also observed.

Norms + feedback. The norms+feedback IHD was coded to display current kW consumption, along with the current $\mathrm{kW}$ consumption of "similar households." The similar households comparison was the average consumption of the other households with the norms+feedback devices. To get the reported consumption rate, participants were given an Eagle Gateway in addition to the IHD. The Eagle Gateway connected wirelessly to the smart meter, and to the Internet through an Ethernet port. The Eagle sent the household's $\mathrm{kW}$ to a cloud server, where it was averaged with the energy use of other households with an Eagle, and then sent back to the Eagle. The IHD would then display the average consumption of similar households in addition to that household's current use. The lights were coded to provide an injunctive norm, which demonstrates the approval or disapproval of the resident's current energy use. If the household was using more than the "similar households"" average, the light displayed red. Similarly, the light displayed green if the household was using less, and yellow if usage was the 
same. The lights were coded using the same $30 \%$ threshold as the others. See Figure 1 for an image of the norms+feedback and Eagle Gateway devices.

Informational video. A short informational video was developed to provide factual information about climate change, and to explicitly link household energy consumption with climate change. The video was a 2-minute animated short that described the ecological effects of electricity consumption on the San Diego Region. At the time of this writing, the video was available online at: http://youtu.be/22H-LnfjnPQ.

Post-survey. At the conclusion of the study, participants were given a follow-up survey. The survey contained the same IHD-relevant survey questions from the pre survey, along with three additional factual knowledge questions specific to the video (for example, "Which fuel do power plants in San Diego County use most to make electricity, coal or natural gas?"), frequency of five possible energy reducing behaviors the participant may have done (for example, "In the past three months how often have you turned off heating or air conditioning at night"), and ten IHD specific questions. The IHD questions were only given to participants who received an IHD, and measured recall of the display, how often they checked the device, if there were any problems with the device, and if the participant would like to keep the device.

\section{Procedure}

Using Google maps and Zillow.com, the team selected 6,500 houses in North County San Diego for possible participation in the study. The physical attributes of each house were recorded, such as presence of a pool, square footage, number of bedrooms and bathrooms, and any solar panels, along with the postal addresses. Following the Tailored Design Method [25], a prenotification postcard was sent to each household followed by the survey a week later. To increase response rates, all addresses were hand written, and a window cling with the university 
logo was included with each survey. The mailing also contained a postage paid return envelope addressed to the university.

Following the initial mailing, researchers went door-to-door to recruit additional participants. Participants were encouraged to fill the survey out at the door, however they were also given the option to mail back the survey in a pre-posted return envelope. As a result of the recruitment efforts, there was an $18 \%$ positive response rate. To be eligible for the study, participants had to indicate on the survey that they had Internet through a modem in their home, and had to provide either an email address or a telephone number. Of the 1157 households agreeing to participate, 816 met the selection criteria for the study and were eligible to participate.

The 816 participants were randomly assigned to 1 of 8 conditions, which were included in the 2 (video, no video) x 4 (feedback only, cost+feedback, norm+feedback, or no contact control) factorial study design. Researchers contacted each participant by phone and arranged to personally deliver and install the IHDs to each household. Participants in the "video control" condition were told that the researchers needed to check the home's smart meter.

Two researchers visited every home dressed in university t-shirts and a nametag. It took approximately four weeks to deploy the in-home displays to participating households, and change was noted for each household starting after its deployment. Researchers placed the IHD in a high-traffic location within the home, such as the kitchen or living room. The researchers informed the participant how the device worked and gave a demonstration. Participants in the video conditions were shown the video on a portable video tablet following the device set up. Participants were informed that researchers would call in three months to complete a short follow up survey over the phone. In total, 431 households were visited or assigned to the control group: feedback (Sample Size $(N)=53$, feedback+video $N=55$, cost+feedback $N=63$, 
cost+feedback+video $N=51$, norms+feedback $N=41$, norms+feedback+video $N=46$, video only $N=59$, no contact control $N=63$ ).

Following the distribution of the IHDs, each household's energy usage was monitored for three months. After three months, participants were contacted by phone to complete the postsurvey. For those participants who could not be reached by phone, a survey and return envelope was delivered to their home. In total, $90 \%$ of the participants completed the post-survey.

Energy use data was provided to the research team directly from the utility. The password protected data files contained hourly kWh consumption for every household in the study for the year prior to the start date through the duration of the study. In addition, hourly temperature and humidity data were taken from the NOAA Mesowest database.

\section{Results}

Statistical analyses were performed using daily household $\mathrm{kWh}$ consumption obtained from the smart meters. The data were obtained in hourly intervals, and then summed to produce daily units. Examination of the distribution revealed 29 households with one or more outlying data points, and these scores were winsorized to 3 standard deviations above the mean for each time point. Across the full sample, the average daily consumption for the two weeks prior to intervention was $23.83 \mathrm{kWh}($ Standard Deviation $(S D)=14.58$; Sample size $(N)=431)$, and the distribution had a positive skew $($ skew $=1.70)$. The analyses reported below were based on average household daily consumption during the two weeks prior to receiving the device, and the week after the delivery date to receiving the device, inclusive of the delivery day. Because households were randomly assigned to treatment, our analyses are based on between-group comparisons during the first week, first month, and 3-month follow-up periods using baseline 
kWh usage as a covariate to control for typical household consumption patterns. The average daily kWh consumption for the four experimental conditions during the baseline week, and then for the two weeks following deployment, is shown in Table 1.

An Analysis of Covariance (ANCOVA) was conducted to test differences in electricity use during the first week of device deployment between the four IHD types (feedback, cost, norms, and control), with baseline use as the covariate. The baseline consumption was a significant covariate, $F(1,426)=779.99, p<.001, \eta^{2}=.64$. The ANCOVA showed that there was a main effect for IHD type, $F(3,426)=2.80, p=.04, \eta^{2}=.01$. Follow up a priori comparisons showed that the norm condition used significantly less electricity during the first week following deployment than the control and the cost condition. The feedback condition was not significantly different than any of the other conditions (all $p$ 's $>.05$ ). These results are illustrated in Figure 2 and Table 2. Compared to the control condition, in the first week following deployment households in feedback condition used 3\% less electricity per day, households in the cost condition used $+0 \%$ more than the control, and households in the norm condition used $9 \%$ less.

Our second analysis tested for the moderating role of the educational video. The analysis was a 4 (IHD type: feedback, cost, norms, or control) by 2 (video, no video) factorial ANCOVA. Again, baseline use was used as a covariate to control for typical household consumption, and average daily electricity use during the first week was the dependent measure. Baseline consumption was a significant covariate, $F(1,422)=754.67, p<.001, \eta^{2}=.64$. Consistent with the analysis in the preceding paragraph, a main effect was found for condition, $F(3,422)=2.71$, $p=.045, \eta^{2}=.02$. However, there was no main effect for video $(F(1,422)=1.56, p=.21)$, or an interaction between treatment and video $(F(3,422)=.37, p=.78)$. Households who watched the 
video $\left(\right.$ Mean $\left(M_{a d j}\right)=24.30$, Standard Error $\left.(S E)=.49\right)$ did not differ significantly in their electricity consumption than those who did not watch the video $\left(M_{a d j}=23.42, S E=.49\right)$.

\section{Treatment Moderators}

The next set of analyses tested our hypotheses about household characteristics that moderated the initial treatment effects. Because our hypothesized household moderators were continuous, we switched to a regression framework for these analyses. For each analysis, we coded treatment as 1, and control as 0 . Electricity usage in the week following device deployment was regressed onto each dummy-coded treatment variable, simultaneously with baseline usage to control for typical household consumption. These analyses replicated our earlier results, showing reduced consumption for households in the norm treatment as compared to control households $(b=-.09, t(206)=-2.40, p=.02)$, and no significant reduction for feedback $(b=-.04, t(227)=-.91, p=.36)$ or cost $(b=.02, t(233)=.47, p=.64)$ as compared to control.

Income. To test the hypothesis that income moderated the relationship between receiving a cost device and electricity use, a regression analysis was conducted. Baseline use was added to the model as a covariate on step one. Device type (cost and control only) and income were centered and then added on step two; the multiplicative interaction term was entered on step three. Baseline use was a significant covariate, $b=.75, t(221)=17.42, p<.001$. Income was a significant predictor of average daily electricity consumption during the first week of deployment, $b=-.09, t(221)=-2.09, p=.04$, however, income was not a significant moderating variable for participants who received the cost device $(p=.72)$.

Motivation. Next, a regression analysis was conducted to test the hypothesis that motivation to use less electricity moderated the relationship between receiving feedback and 
electricity consumption. Motivation to use less electricity was assessed with four questions on the pre-survey (discussed previously as obligation to use less electricity). Motivation was calculated by averaging responses to these questions. Baseline use was added to the model as a covariate. Device type (feedback=1 and control=0), motivation to use less electricity, and the multiplicative term were centered and added to the model. Baseline use was a significant covariate, $b=.82, t(225)=21.02, p<.001$. Motivation to reduce energy use and feedback device were both not significant predictors of energy use during the first week of the intervention $(p$ $=.34$ and $p=.38$, respectively). The multiplicative term was also not significant $(p=.61)$.

Climate change knowledge. The next regression analysis tested the hypothesis that knowledge about climate change would moderate the relationship between receiving a normative device and electricity consumption. Climate change knowledge was assessed with three questions, which were averaged to produce a mean response. Baseline electricity use was added to the model as a covariate. Device type (norm=1 and control=0), mean score for climate change knowledge, and the multiplicative term were centered and added to the model. Baseline use was a significant covariate, $b=.83, t(201)=21.01, p<.001$. Device type was a significant predictor in the model, $b=-.09, t(201)=-2.24, p=.03$. However climate change knowledge and the multiplicative effect were not significant ( $p=.36$ and $p=.29$, respectively).

Baseline usage. Finally, a regression analysis was conducted in order to test if baseline usage moderated the relationship between device type and electricity consumption. Baseline use was computed by averaging the daily electricity consumed for each household for the two weeks prior to device deployment. Separate regression equations were computed for each intervention compared to the control condition. Device type, mean baseline use, and the multiplicative term were added to the model for each equation. Average baseline use was a significant predictor, $b$ 
$=.86, t(226)=16.02, p<.001$. Device type and the multiplicative term were both not significant predictors ( $p=.43$ and $p=.20$, respectively). Similar results were found for the cost and the norm conditions, and in neither condition was there a significant interaction between device type and baseline consumption ( $p=.35$ for the cost condition, and $p=.38$ for the norm condition).

\section{Three Month Energy Use}

Data were analyzed for 393 households that had complete data through the 3 months of the study. In order to determine long-term reductions over the course of the study, energy use was calculated as percentage change between baseline and the three-month average energy use for each condition. Changes in energy use can be seen in Figure 4. As shown in the Figure, the control condition used $8.34 \%$ more electricity in the three months following deployment of the device, most likely due to seasonal effects. The feedback condition used $9.15 \%$ more than baseline, but did not differ from the control. Similarly, the cost condition used $7.21 \%$ more than baseline, but did not differ significantly from the control. The norm condition used only $1.32 \%$ more than baseline, and the change was significantly different from the control and feedback conditions $(p<.05)$. The difference between the change in consumption for the control condition and the change for the norm condition was 7.02\%, which reflects the 3-month effect of the norms IHD.

\section{Post-Survey}

Three hundred and eighty of a potential 424 participants (90\%) responded to the postsurvey. Three factual questions were asked to assess knowledge about climate change. The answers to these questions were presented in the video that was shown to half of the participants. Responses were coded as correct $=1$ and incorrect $=0$, and knowledge scores ranged from 0 to 3 . An ANOVA was used to test if those who watched the video were more knowledgeable than those who did not watch the video. Results showed that residents who watched the video $(M=$ 
2.63, $S D=.58, N=157$ ) answered significantly more questions correctly than those who did not watch the video $(M=2.47, S D=.65, N=144 ; t(299)=2.24, p=.03, d=.26)$. There were no differences in knowledge about climate change at the follow-up across the four experimental IHD conditions.

Two separate measures served as manipulation checks and to test the educational impact of the IHDs. First, participants were asked four questions about their knowledge of electricity consumption in their homes. Responses to the four questions were averaged to produce an overall measure of knowledge. The same questions were asked on both the pre- and post-surveys. A paired samples t-test showed there were no significant differences between the original survey and the post-survey in reported knowledge of household electricity consumption for any of the conditions. See Table 3. Secondly, participants were asked in an open-ended question to describe the information displayed on their device. Overall, $74.6 \%$ of participants answered the openended question correctly. Correct answers were given by $83.8 \%$ of participants in the feedback condition, $63 \%$ in the cost condition, and $77.6 \%$ in the norm condition. Participants did not differ significantly in the number of times per day they reported looking at their device $(M=6.85, S D$ $=6.47, N=277 ; F(2,274)=.01, p=.99)$.

A 2 (pre survey, post-survey) by 4 (feedback, cost, norm, control) mixed model ANOVA tested the differences in participants' reported obligation to use less electricity between the original and follow-up survey. By analyzing differences between responses on the pre-survey and post-survey by condition, this analysis tests the differential response between the within subjects variable (time) and between subjects variable (IHD condition) on reported obligation to use less electricity. There was a significant interaction between IHD condition and pre-post $(F(3$, $\left.376)=3.42, p=.02, \eta^{2}=.03\right)$. Follow-up pairwise comparisons showed that participants in the 
feedback condition and participants in the cost condition reported a higher obligation to use less electricity on the post-survey than on the original survey. There were no significant differences for the norm or control condition. See Table 3. A similar analysis was performed on the rated degree to which the device motivated the participants to use less electricity. Results showed no significant differences across conditions $(M=5.63, S D=1.62, N=284)$.

Five questions were used to assess behavior changes that occurred during the study (e.g., installing energy efficient light bulbs, limiting shower length). Responses on the reported frequency of five behaviors were averaged to produce a scale score. A oneway ANOVA using the mean of the five questions showed that there were no significant differences in reported behaviors between the conditions $(p>.05)$.

Finally, participants were offered to keep their device or to have it picked up. Of the 281 participants with a device who completed the follow up survey, 13.7\% returned their device. The fewest returns were from those in the cost condition (6\%), second most were from those with feedback devices $(13.1 \%)$, and the most returned were by those in the norm condition (23.5\%). When asked how accurate their device was, participants with norm feedback devices perceived their IHDs as significantly less accurate than those with a feedback device or those with a cost device. See Table 3.

\section{Discussion}

The results from the experiment reported in this paper show that feedback provided through in-home displays (IHDs) can affect residential electricity consumption. First, the results showed that households receiving simple feedback of $\mathrm{kW}$ consumption did not differ from a randomized control group in either the short-term (1-week following deployment) or longer-term 
time frame (3-months following deployment). Similar results were found for cost-framed feedback, but the norm-framed feedback condition showed a significant reduction in consumption at both the short-term and longer-term periods. The results from the reported experiment are in-line with previous studies demonstrating that normative feedback can be an effective tool for promoting residential electricity conservation, and that in-home displays offer a promising medium for presenting such feedback $[11,12,24]$. The findings illustrate the importance of message frame, and demonstrate that feedback alone is not sufficient to alter behavior. In-line with previous studies, feedback works best when coupled with a motivational goal [14].

In the current study, we did not modify the pricing or payment process associated with electricity consumption, and as a result, the cost-frame failed to affect consumption. Given the ubiquity of cost-framed messages in the energy domain, we want to offer some discussion of the lack of effects. The IHDs that were used in this experiment were coded to display instantaneous $\mathrm{kW}$ consumption and projected hourly cost if consumption continued at that rate. However due to the hourly frame, these rates were typically low. For example, the average household used $23.83 \mathrm{kWh}$ per day during the baseline period. This translates into an hourly rate of $.99 \mathrm{kWh}$ over the 24-hour period. Based on the tiered rate structure at the utility, the highest tier was 39cents per kWh (standard tier 4, summer rates). To illustrate, a typical home would see a message displayed that they were using $.99 \mathrm{~kW}$, and this was costing them 36-cents per hour. This appears relatively low, despite the fact that this rate of consumption would generate a monthly electricity bill of $\sim 233$.

Our results showed that the norm message frame had the largest impact on electricity consumption. In the initial two weeks following deployment, households with the norm-framed 
IHD used 9\% less electricity than did the households in the control condition, and $7.02 \%$ less in the next three months. This effect is consistent with previous studies $[17,20,23]$, and shows that the norm-based frame offers a solid alternative to cost-based message framing.

The study also tested the impact of climate change educational materials, both in isolation and as a complement to feedback. Findings from the experiment did not provide any evidence that the educational materials were effective at reducing consumption. Across all three conditions, households that received the educational materials did not differ in their electricity consumption from households that did not view the educational video. However, results from the post-survey did show an impact on knowledge — households that viewed the educational video were significantly more knowledgeable about climate change three months after viewing the video compared to households that did not. These findings reinforce previous research showing that knowledge is neither necessary nor sufficient to produce conservation [7,26,27].

Finally, in terms of customer engagement, the results showed that residents liked and used the IHDs. Residents reported looking at the IHD an average of 6.85 times per day, and there were no differences across the experimental groups. When given the opportunity to keep the IHD, $85 \%$ asked to keep it. Interestingly, more residents in the cost condition (94\%) and the feedback condition (87\%) asked to keep the device, compared to the norm condition (76\%). Similarly, residents in the cost and feedback conditions reported that the IHD encouraged them to use less electricity, compared to the norm condition — this effect despite the fact that they did not use significantly less electricity. These findings are aligned with previous studies showing that the influence of normative messages is often underdetected [20,28,29].

In conclusion, the results from the reported study show that in-home displays can be an effective tool for reducing residential demand for electricity, however the effectiveness of the 
displays depends on the frame provided for the feedback. In a randomized control trial with 431 single-family households, we found that a norm-based message frame was effective at reducing residential demand, but that simple feedback or feedback framed as cost was not effective at reducing demand. As new technologies become available for providing consumers with enhanced feedback, it is important to connect these communication tools to behavioral science, and to test their impact using randomized controlled designs.

\section{References}

[1] Edison Foundation. Utility-scale smart meter deployments, plans, and proposals. Washington, DC: Institute for Electric Efficiency 2012.

[2] Navigant. Smart electric meters, advanced metering infrastructure, and meter communications: Global market analysis and forecasts. Washington, DC: Navigant Research 2014. http://www.navigantresearch.com/.

[3] Balista NC, Melício R, Matias JCO, Catalão JPS. Photovoltaic and wind energy systems monitoring and building/home energy management using ZigBee devices with a smart grid. Energy 2013;49:306-15.

[4] Sintov ND, Schultz PW. Unlocking the potential of smart grid technologies with behavioral science. Frontiers in Psychology. Special Issue on Understanding the Human Factor of the Energy Transition: Mechanisms Underlying Energy-relevant Decisions and Behavior. 2015;6.

[5] Armel KC, Gupta A, Shrimali G, Albert A. Is disaggregation the holy grail of energy efficiency? The case of electricity. Energy Policy 2013;52:213-34. 
[6] Ehrhardt-Martinez K, Donnelly K, Laitner J. Advanced Metering Initiatives and residential feedback programs: A meta-review for household electricity-saving opportunities. Washington, D.C.: American Council for an Energy-Efficient Economy 2010. http://www.aceee.org/research-report/e105

[7] Abrahamse W, Steg L, Vlek C, Rothengatter T. A review of intervention studies aimed at household energy conservation. Journal of Environmental Psychology 2005;25(3):273-90.

[8] Kluger A, DeNisi A. The effects of feedback interventions on performance: a historical review, a meta-analysis, and a preliminary feedback intervention theory. Psychological Bulletin 1996;119:254-84.

[9] Charles D. Leaping the efficiency gap. Science 2009;325:804-11.

[10] Darby S. The effectiveness of feedback on energy consumption: A review for DEFRA of the literature on metering, billing, and direct displays. Environmental Change Institute: Oxford. 2006. http://www.defra.gov.uk/ENVIRONMENT/climatechange/uk/energy/ research/pdf/energyconsump-feedback.pdf.

[11] Electric Power Research Institute. Residential electricity use feedback: A research synthesis and economic framework. Palo Alto, CA. 2009.

[12] Fischer C. Feedback on household electricity consumption: a tool for saving energy? Energy Efficiency 2008;1:79-104.

[13] Katzev R, Johnson T. Promoting energy conservation: An analysis of behavioral research. Boulder, Colorado: Westview press 1987.

[14] Locke E, Latham G. Building a practically useful theory of goal setting and task motivation, a 35-year odyssey. American Psychologist 2002;57:705-17. 
[15] Schultz PW. Making energy conservation the norm. In K. Ehrhardt-Martinez \& J. Laitner (Eds.), People-centered initiatives for increasing energy savings. Washington, DC: American Council for an Energy-Efficient Economy. 2010.

[16] Delmas M, Fischlein M, Asensio O. Information strategies and energy conservation behavior: A meta-analysis of experimental studies from 1975 - 2011. Energy Policy 2013;61:729-39.

[17] Schultz PW, Nolan JM, Cialdini RB, Goldstein NJ, Griskevicius V. The constructive, destructive, and reconstructive power of social norms. Psychological Science 2007;18(5):429-34.

[18] Costa DL, Kahn ME. Energy conservation "nudges” and environmentalist ideology: Evidence from a randomized residential electricity field experiment. Journal of the European Economic Association 2013;11(3):680-702.

[19] Froehlich J. Promoting energy efficient behaviors in the home through feedback: The role of human-computer interaction. HCIC2009 Winter Workshop. 2009. http://www.cs.umd.edu/ jonf/publications/Froehlich_PromotingEnergyEfficientBehavirosI nTheHomeThroughFeedback-TheRoleOfHumanComputerInteraction_HCIC2009.pdf

[20] Nolan J, Schultz PW, Cialdini RB, Griskevicius V, Goldstein N. Normative social influence is underdetected. Personality and Social Psychology Bulletin 2008;34:913-23.

[21] Alberini A, Gans W, Velez-Lopez D. Residential consumption of gas and electricity in the U.S.: The role of prices and income. Energy Economics 2011;33(5):870-81.

[22] Espey JA, Espey M. Turning on the lights: A meta-analysis of residential electricity demand elasticities. Journal of Agricultural and Applied Economics 2004;36:65-81.

[23] Allcott H. Social norms and energy conservation. Journal of Public Economics 
2011;95:1082-95.

[24] Faruqui A, Sergici S, Sharif A. The impact of informational feedback on energy consumption-a survey of the experimental evidence. Energy 2010;35:1598-608.

[25] Dillman DA. Mail and Internet surveys: The tailored design method. Hoboken, NJ:Wiley. 2007.

[26] Bolderdijk JW, Steg L, Geller ES, Lehman PK, Postmes T. Comparing the effectiveness of monetary versus moral motives in environmental campaigning. Nature Climate Change $2013 ; 3: 413-6$.

[27] Schultz PW. Knowledge, education, and household recycling: Examining the knowledgedeficit model of behavior change. In T. Dietz \& P. Stern (Eds.), New tools for environmental protection. Washington DC: National Academy of Sciences 2002;67-82.

[28] Cialdini RB. Basic social influence is underestimated. Psychological Inquiry 2005;16:15861.

[29] Nolan J, Kenefick J, Schultz PW. Normative social influence is underdetected by energy experts... unless you show them the data. Social Influence 2011;6:169-80. 
Table 1: Average daily electricity consumption for households in the four experimental conditions

\section{Mean kWh per day Standard Deviation (SD)}

\begin{tabular}{llll}
\hline Feedback IHD & Baseline Use & 21.37 & 9.78 \\
$\mathbf{N = 1 0 8}$ & Week One & 23.00 & 10.88 \\
& Week Two & 24.61 & 12.81 \\
\hline $\mathbf{C o s t}$ IHD & Baseline Use & 23.15 & 10.01 \\
$\mathbf{N = 1 1 4}$ & Week One & 26.02 & 12.53 \\
& Week Two & 26.78 & 13.12 \\
\hline Norm IHD & Baseline Use & 22.13 & 10.72 \\
$\mathbf{N}=\mathbf{8 7}$ & Week One & 22.30 & 11.88 \\
& Week Two & 23.28 & 12.14 \\
\hline $\mathbf{C o n t r o l}$ & Baseline Use & 21.65 & 9.39 \\
$\mathbf{N = 1 2 2}$ & Week One & 24.07 & 11.96 \\
& Week Two & 24.92 & 11.76 \\
\hline
\end{tabular}


Table 2: Statistical results for each condition compared to the norm condition one week following device deployment

\begin{tabular}{|c|c|c|c|c|c|}
\hline \multirow[t]{2}{*}{ Condition } & \multirow[t]{2}{*}{$M_{a d j}{ }^{\mathbf{a}}$} & \multirow[t]{2}{*}{$S E^{\mathbf{a}}$} & \multicolumn{3}{|c|}{ Comparison to Norm } \\
\hline & & & $t^{\mathrm{b}}$ & $p$ & $d$ \\
\hline $\begin{array}{l}\text { Feedback } \\
(\mathrm{N}=108)\end{array}$ & 23.67 & .68 & -1.39 & $>.05$ & .20 \\
\hline $\begin{array}{l}\text { Cost } \\
(\mathrm{N}=114)\end{array}$ & 24.99 & .66 & -2.73 & $.01^{\mathrm{c}}$ & .39 \\
\hline $\begin{array}{l}\text { Norm } \\
(\mathrm{N}=87)\end{array}$ & 22.25 & .76 & & - & \\
\hline $\begin{array}{l}\text { Control } \\
(\mathrm{N}=122)\end{array}$ & 24.48 & .64 & -2.26 & $.02^{\mathrm{c}}$ & .32 \\
\hline
\end{tabular}
a. Mean and standard error $(S E)$ adjusted for baseline consumption covariate
b. Values reported from the $t$-computations and effect sizes $(d)$ are based on the pooled MSE from the ANCOVA $(M S E=49.64, d f=426)$.
c. Differences statistically significant at $\mathrm{p}<.05$ 
Table 3: Statistical results from pre- to post-survey comparisons across the four experimental conditions

\begin{tabular}{|c|c|c|c|c|c|c|c|c|c|}
\hline \multirow[t]{2}{*}{ Condition } & \multicolumn{3}{|c|}{$\begin{array}{c}\text { Knowledge of } \\
\text { household energy use }\end{array}$} & \multicolumn{3}{|c|}{$\begin{array}{l}\text { Obligation to use less } \\
\text { electricity }\end{array}$} & \multicolumn{3}{|c|}{$\begin{array}{l}\text { Perceived accuracy of } \\
\text { IHD device }\end{array}$} \\
\hline & $M_{\text {diff }}^{\mathrm{a}}$ & $S D$ & $N$ & $M_{\text {diff }}$ & $S E$ & $N$ & $M$ & $S D$ & $N$ \\
\hline Feedback & .08 & 1.68 & 99 & .22 & .11 & 99 & 5.94 & 1.41 & 99 \\
\hline Cost & .20 & 1.40 & 99 & .30 & .11 & 100 & 5.83 & 1.38 & 99 \\
\hline Norm & .27 & 1.51 & 84 & .07 & .12 & 84 & 5.18 & 1.84 & 85 \\
\hline Control & .08 & 1.56 & 96 & .15 & .12 & 96 & & & \\
\hline
\end{tabular}

a. $\quad M_{\text {diff }}=$ mean difference in responses between pre and post surveys; $S D=$ standard deviation; $N=$ sample size; $S E=$ standard error; $M=$ mean response

b. Participants in the feedback condition $(t(98)=2.08, p<.05, d=.30)$ and participants in the cost condition $(t(99)=2.83, p<.05, d=.40)$ differed significantly from those in the norm and control conditions.

c. Participants in the norm condition perceived their device as significantly less accurate than those with a feedback device $(t(181)=3.42, p=.001, d=.51)$ or those with a cost device $(t(181)=2.94, p=.003, d$ $=.44)$. 
Figure 1: Examples of Three Smart Meter-Connected In-Home-Displays
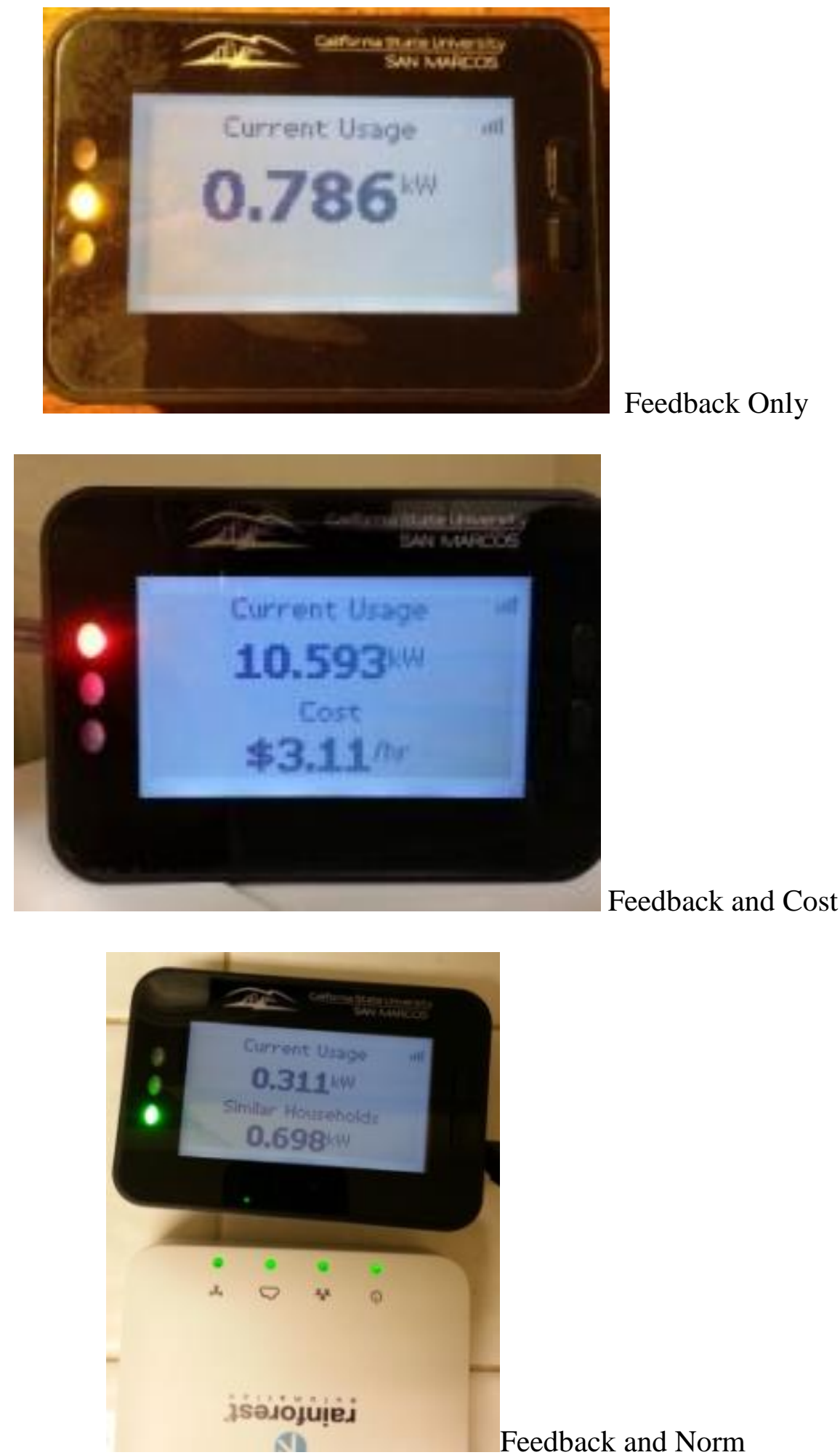
Figure 2: Average Daily Electricity Consumption During the First Week Following Deployment of In-Home Displays

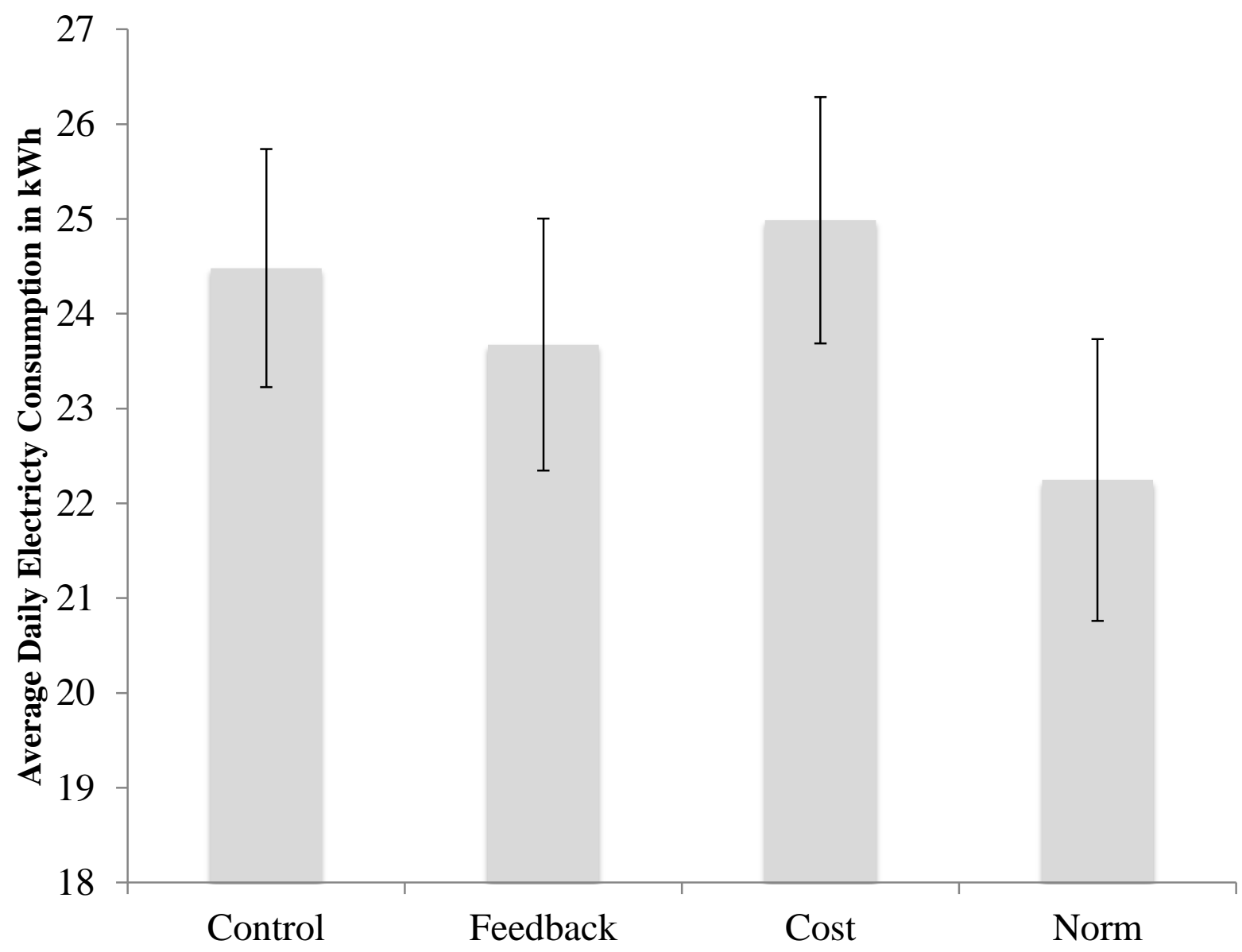

Note: Error bars represent $95 \%$ confidence intervals around the adjusted mean. The norm condition was significantly different from the control $(p<.05)$ and the difference in electricity consumption over the initial 1-week follow-up period was $9 \%$. 
Figure 3: Average Daily Electricity Consumption by Week for Three Months Following Deployment of In-Home Displays

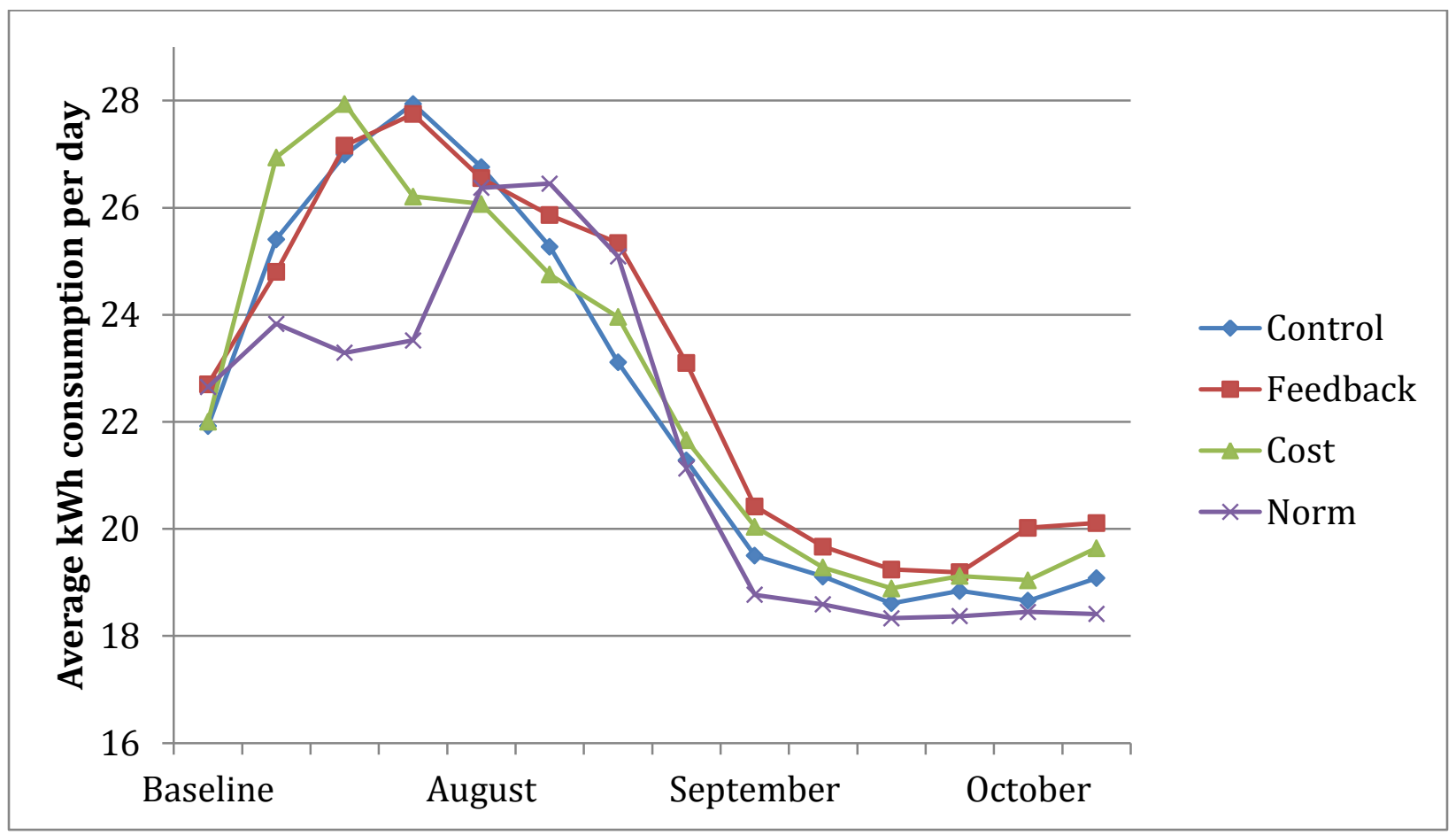

Note: Only households with three complete months of follow-up data are included in the figure. Baseline represents the two weeks prior to deploying the IHDs, so all subsequent data points reflect the impact of the IHD. The labels for the months shown in the $\mathrm{x}$-axis are approximate, as deployment lasted four weeks and not all houses received the IHD on the same date. 
Figure 4: Average Daily Electricity Consumption Change from Baseline During the Three Months Following IHD Deployment

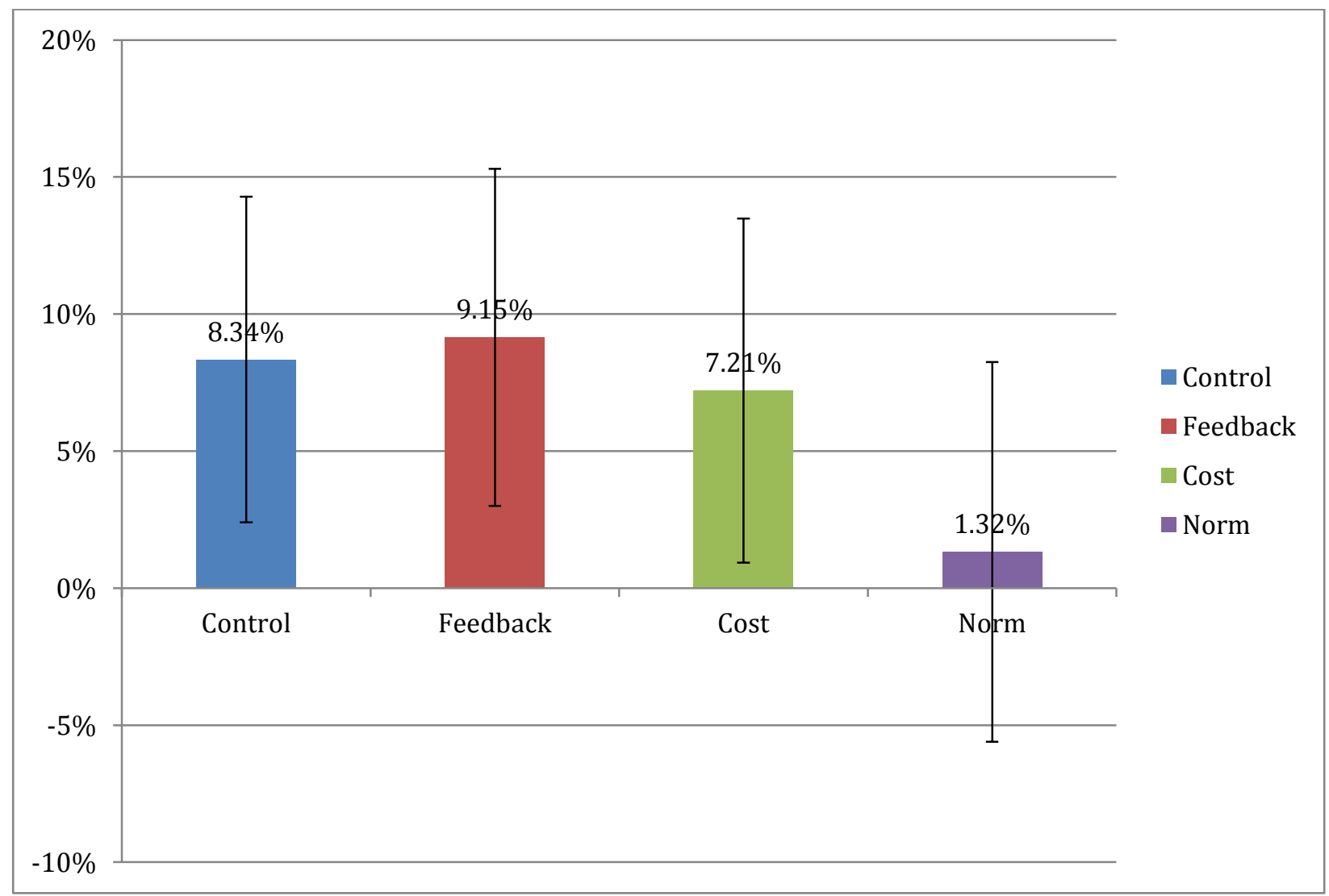

Note: Error bars represent 95\% Confidence Intervals around the mean. The norm condition was significantly different from the control condition $(p<.05)$, and the difference in electricity consumption over the 3 -month follow-up period was $7.02 \%$. 